\title{
Effect of Brain Derived Neurotrophic Factor, In Relation to Diet and Life- style Factors, for Prevention of Neuropsychiatric and Vascular Diseases and Diabetes
}

\author{
Ram B. Singh ${ }^{1, *}$, Toru Takahashi ${ }^{2}$, Miki Tokunaga ${ }^{3}$, Agnieszka Wilczynska ${ }^{1}$, Chee J. Kim ${ }^{4}$, \\ Fabien De Meester ${ }^{1}$, Teodora Handjieva-Darlenska ${ }^{5}$, Sukhinder K. Cheema ${ }^{6}$, Douglas W. Wilson ${ }^{7}$, \\ Branislav Milovanovic ${ }^{8}$, Jan Fedacko ${ }^{9}$, Krasimira Hristova ${ }^{10}$ and Hilton Chaves $^{11}$
}

\begin{abstract}
${ }^{I}$ The Tsim Tsoum Institute, Krakow, Poland; ${ }^{2}$ Graduate School of Human Environment Science, Fukuoka Women's University, Japan; ${ }^{3}$ Department of Nutrition and Health Sciences, Fukuoka Women's University, Fukuoka, Japan; ${ }^{4}$ Korean Society of Lipidology and Atherosclerosis, Seoul, Korea; ${ }^{5}$ Department of Pharmacology, Medical University in Sofia, Bulgaria; ${ }^{6}$ Department of Biochemistry, Faculty of Medicine, Memorial University of Newfoundland, St. John's, NL, Canada; ${ }^{7}$ School of Medicine, Pharmacy and Health, Durham University, UK; ${ }^{8}$ Neurocardiological Laboratory, Department of Cardiology, University Clinical Center Bezanijska Kosa, Medical Faculty, University of Belgrade, Serbia; ${ }^{9}$ PJ Safaric University, Kosice, Slovakia; ${ }^{10}$ National Heart hospital, Sofia, Bulgaria; ${ }^{11}$ Federal University of Pernambuco, Recefee, Brazil
\end{abstract}

\begin{abstract}
Background: Brain-derived neurotrophic factor (BDNF) is a major neurotrophin which may have promise to be a nutraceutical of this decade. It has a documented role in neurogenesis, angiogenesis, and neuronal survival. BDNF can have beneficial effects on several cardio-metabolic and neuro-psychiatric disorders, indicating that it is important in brainbody interactions. Diet and lifestyle factors may also have an influence on BDNF levels. In this review, we examine the beneficial role of BDNF on risk factors of vascular diseases, type 2 diabetes mellitus and anxiety disorders. Methods: Internet search and discussion with peer colleagues. Results: Majority of the BDNF (70-80\%) is derived from dendrite of neurons but it is also present in other body tissues. BDNF controls the food intake and appetite as well as lipid and glucose metabolism. Sedentary behavior and tobacco intake may be associated with BDNF deficiency. Lower serum concentration of BDNF and higher vascular endothelial growth factor (VEGF) concentrations were associated with increased risk of incident stroke/TIA. BDNF may serve as an intermediate biomarker for subclinical vascular disease and may also have biological potential to serve as a therapeutic target for primary and secondary prevention of vascular diseases, as well as clinical and subclinical vascular brain disease. BDNF deficiency has been observed in association with anxiety, depression, insomnia, dementia, insulin resistance, type 2 diabetes and vascular diseases. The phenotypes associated with insulin resistance are at increased risk for developing cognitive decline and neuro-degeneration resulting in vascular dementia, and depression as well as diabetes mellitus and metabolic syndrome, which are risk factors for CVDs. BDNF may be administered as nutraceutical due to its protective influence on BDNF concentrations, insulin receptors and hypothalamic dysfunction leading to beneficial effects on cardiovascular risk and neuropsychological dysfunction. It is proposed that omega-3 fatty acids and moderate physical activity may enhance BDNF release. Conclusions: It is possible that circulating BDNF deficiency is a risk factor for obesity, CVDs and diabetes as well as risk factor for neuropsychiatric diseases. BDNF administration may modify the risk of clinical and subclinical stroke, depression, and dementia as well as of obesity and type 2 diabetes.
\end{abstract}

Keywords: Degenerative diseases, depression, obesity, type 2 diabetes, vascular disease.

\section{INTRODUCTION}

Nutritional deficiencies and appreciable overnutrition in the form of obesity and metabolic syndrome are common in most countries [1,2]. These metabolic problems may have a route via brain-body interactions [3-10]. Western style foods rich in refined carbohydrates, linoleic acid, saturated and total fat and low dietary n-3 fatty acids and flavonoids, in conjunction with physical inactivity, anxiety and depression can predispose inflammation leading to cardiovascular diseases (CVDs) [1-7]. An experimental study showed that

*Address correspondence to this author at the Halberg Hospital and Research Institute, Civil Lines, Moradabad (UP), India;

Tel: 0091591 2417437; Email: rbs@tsimtsoum.net vagal nerve stimulation attenuated the systemic inflammatory response to endotoxin indicating that brain appears to have significant influence on biological dysfunctions in other body systems [8]. Increased sympathetic activity, in presence of lower parasympathetic activity, with increased release of neurotransmitters; catecholamine, cortisol, and serotonin can cause oxidative stress, which may damage the arcuate nucleus as well as the pro-opiomelanocortin (POMC) neurons in the hypothalamus and macrophages, and the liver and may release pro-inflammatory cytokines, which appear to be underlying mechanisms of neuro-cardiovascular risk [4-10].

It is possible that dietary long chain polyunsaturated fatty acid (PUFA), flavonoid and coenzyme Q10 deficiency, sedentary behavior and tobacco may inhibit the release of brain- 
derived neurotrophic factor (BDNF) and endocannabinoid receptors in the brain and other body systems; liver, heart, blood vessels, small intestine and pancreas $[9,10]$. BDNF has been implicated in the pathogenesis of major depression [10]. Subjects with type 2 diabetes have a high prevalence of major depression and low levels of BDNF which poses the possibility that the BDNF Val66Met polymorphism may be associated with co-morbid depression and the low serum levels of BDNF which has an important role in regulating maintenance, growth and survival of neurons [10].

Of the total fatty acids in the brain, $30 \%-50 \%$ are long chain PUFA, which are incorporated in the cell membrane phospholipids of neurons and other body cells. It is possible that omega-3 fatty acids, coenzyme Q10 and flavonoids as well as moderate exercise training, that are known to enhance parasympathetic activity and increase the secretion of anti-inflammatory cytokines interleukin IL-4 and IL-10 as well as acetylcholine in the hippocampus, may be protective. It has been demonstrated that biological functions of the human body have a periodicity which is controlled by interactions of solar activity and neuro-endocrine system [11]. It is proposed that BDNF levels also may have periodicity showing lower levels in the morning with increase in the day as a compensatory mechanism. Neurotrophins are considered to play a major role in neural function including survival, development, and plasticity $[10,12]$. This review reports the scientific evidence available on neuro-hormonal and environmental triggers of BDNF in relation to CVDs, anxiety disorders and type 2 diabetes.

\section{CARDIOVASCULAR DISEASE, DIABETES MELLI- TUS AND DEPRESSION}

Epidemiological studies indicate that approximately 26$30 \%$ of patients with type 2 diabetes, suffer from various grades of depression, which are above that of the normal population $[13,14]$. Type 2 diabetes and depression are two of the highly prevalent non-communicable diseases (NCDs) and are major risk factors for CVDs. All these causes and CVD mortality rates are significantly higher among patients with type 2 diabetes and depression than among patients with diabetes without depression [15]. Therefore, it is important to study the etiology of depression with CVDs and type 2 diabetes mellitus. In a randomized controlled trial among 584 patients, aged 60-74 years, of a depression treatment program for older adults based on primary care (PROSPECT), treatment showed beneficial effects on diabetes, depression, and death [16]. Of the 584 participants, 123 $(21.2 \%)$ reported a history of diabetes, who were assessed at 5 years. After a median follow-up of 52.0 months, 110 depressed patients had died. Depressed patients with diabetes in the intervention category were less likely to have died during the 5-year follow-up interval than depressed diabetic patients in usual care after accounting for baseline differences among patients (adjusted hazard ratio 0.49 [95\% CI $0.24-0.98]$ ). It is possible that elderly depressed primary care patients with diabetes, implementing depression care management were less likely to die over the course of a 5-year interval than depressed patients with diabetes in usual-care.

\section{BRAIN DERIVED NEUROTROPHIC FACTOR}

BDNF is the most abundant neurotrophin in the mammalian central nervous system, which protects the neurons from injury, at least in part, by inhibiting apoptosis and by stimulating sprouting and neuronal reorganization. Majority of the BDNF $(70-80 \%)$ is derived from brain but it is normally found in the liver, pancreas, small intestine and arterial cells, indicating its role in the brain-body connections. The synaptic plasticity and survival of neurons may be modulated by BDNF concentration [12]. BDNF appears to be involved in the pathogenesis of several cardio-metabolic and neuropsychiatric disorders, indicating that it is important in brainbody interactions [10-17]. In a recent meta-analysis, a total of 1179 participants were included [12]. The findings showed lower BDNF levels in individuals with any anxiety disorder compared to those without. However, it was dependent on source of BDNF protein and type of anxiety disorder indicating that BDNF concentrations may be decreased in subjects with an anxiety disorder. This observation is not consistent across various anxiety disorders and may largely be explained by the significantly lowered BDNF levels found in depression. BDNF plays a key role in regulating survival, growth and maintenance of neurons [17]. It is possible that reduction in BDNF expression is a pathogenic factor common to Alzheimer's disease, dementia and major depression which are in turn risk factors for CVDs and type 2 diabetes [17-19]. BDNF could be a biomarker of insulin resistance, diabetes mellitus and cardiovascular as well as anxiety and depression, hence it would be useful to understand its role in these disorders $[10,13,14,17-21]$. BDNF metabolism and synthesis are shown in (Fig. 1).

\section{BDNF AND DEPRESSION}

A decrease in serum levels of mature Brain-Derived Neurotrophic Factor, but not its precursor proBDNF, in patients with major depressive disorder has been observed indicating its role in depression [10]. Several workers, have reported that patients with major depression, have significantly lower concentrations of serum BDNF compared with normal controls [20, 21]. However, treatment with antidepressants led to an up-regulation of BDNF in the hippocampus of subjects with major depression [19]. Experimental study indicates that infusion of recombinant BDNF exerted antidepressant effect which confirms the association of BDNF with depression [22]. The direct metabolic role of BDNF is supported by studies on BDNF-deficient mice, which developed diabetes and obesity in early adulthood [23]. It has been reviewed that animals having BDNF deficiency, also have important influence on the regulation of eating behavior and modulation of the secretion and activities of insulin, leptin, ghrelin and proinflammatory cytokines associated with energy homeostasis (Fig. 2) [24-26]. Epidemiologic studies also indicate that patients with type 2 diabetes are at greater risk of depression in association with lower serum BDNF levels compared to non-diabetic individuals [24, 27-29]. This finding poses the possibility that the higher rate of depression in diabetes may be in part mediated by decreased BDNF levels.

\section{BDNF POLYMORPHISM}

The clinical significance of the effect of BDNF levels on depression and the impact of Val66Met polymorphism remain unclear in type 2 diabetes patients with depression. It seems likely that BDNF genetic studies of patients with and without depression may shed light on the potential genetic link between depression and type 2 diabetes [27-36]. It has 


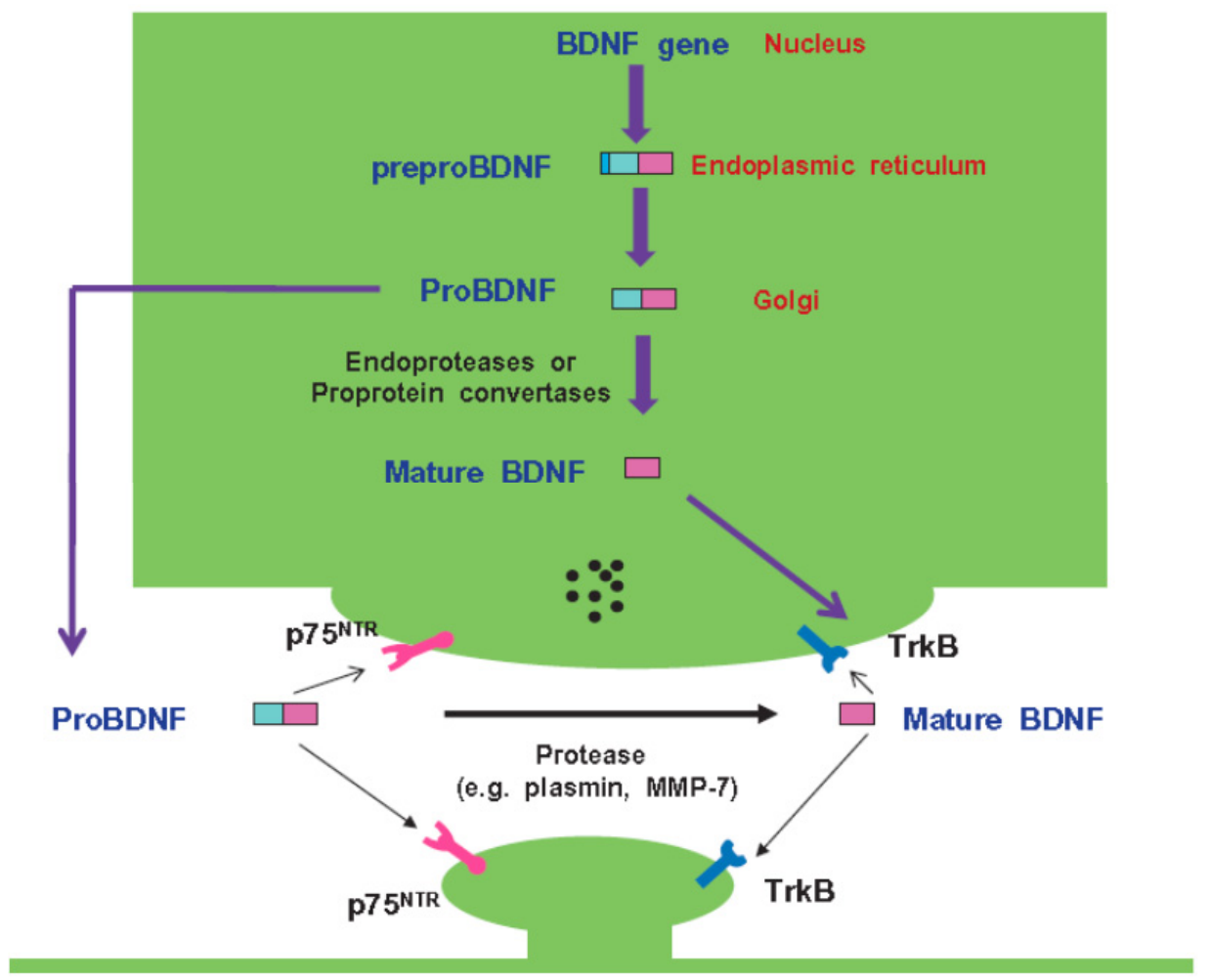

Fig. (1). BDNF metabolism from proBDNF to mature BDNF.

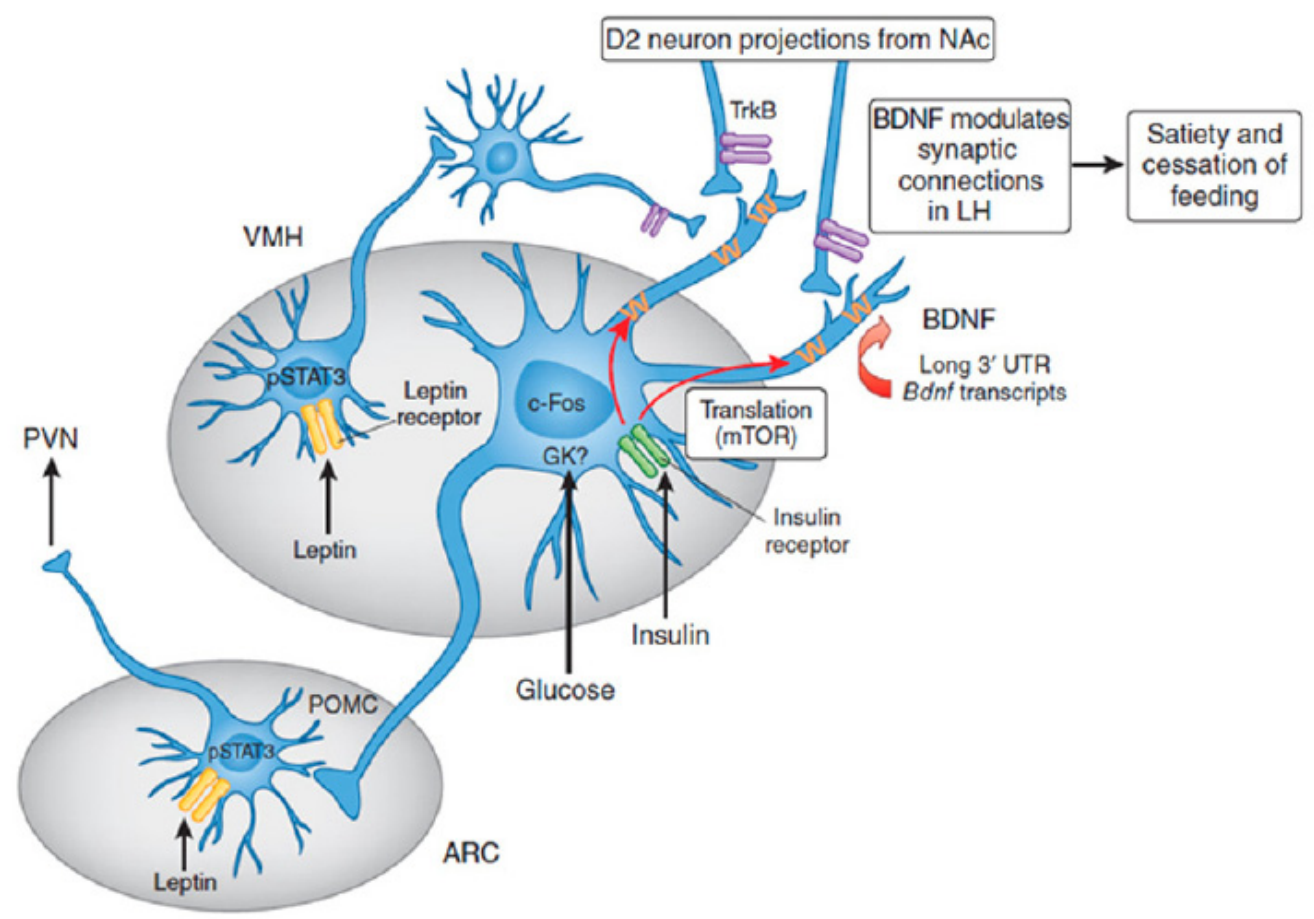

Fig. (2). Mechanism of action of BDNF on hypothalamus, POMC neuron and control of appetite and overeating.

been proposed that a genetic variation (Val to Met substitution) in BDNF, leading to a reduction in serum BDNF levels in type 2 diabetes, may contribute to depression and diabetes [37]. This study examined the association of BDNF Val66Met polymorphism with depression in T2DM of Chinese Han subjects (major ethnic group in East Asia) [37]. There is a positive association between BDNF Val66Met polymorphism and comorbid depression in patients with type 2 diabetes and Met allele carriers are susceptible to suffer from depression. Serum BDNF levels are decreased in diabetes patients carrying either 66Met/Met homozygote or $66 \mathrm{Val} / \mathrm{Met}$ heterozygote compared to those carrying $66 \mathrm{Val}$ homozygote. 
Several studies have demonstrated that the degree of therapeutic response to antidepressants is associated with the BDNF Val66Met polymorphism, suggesting that the BDNF gene may be a good candidate gene for the pharmacogenetic study of antidepressants [18]. The human BDNF gene has been mapped to chromosome $11 \mathrm{p} 13$ and a common single nucleotide polymorphism (SNP). It consists of a missense change (G196A), which produces a valine (Val) to methionine (Met) alteration, that has been identified in the coding exon of the BDNF gene at position 66 (Val66Met) [30]. The intracellular trafficking and activity-dependent secretion of BDNF may depend on Val66Met polymorphism in the BDNF gene [31]. The Met allele may be associated with decline in hippocampal volume in both normal and depressed patients and with decreased executive function and cognition [31, 32]. BDNF Val66Met polymorphism and lower levels of BDNF have been associated with major depressive disorders among Caucasians [33]. Further clinical studies have demonstrated that the Met allele was more commonly observed among individuals with mood disorders [34-36]. However, a higher incidence of depression in Val, not Met carriers has also been observed [38]. Since both alleles have been associated with different disease processes, including emotion response [39], esophageal hypersensitivity [40], individual's sexual activities [41] and depression, hence the effects on brain function remain elusive [33, 42, 43]. An experimental study confirmed that a variant BDNF mouse (BDNF Met/Met) reproduced the phenotypic hallmarks of humans with this variant allele, and exhibited increased anxiety-related behaviors [44].

\section{BDNF AND INSOMNIA}

Slow-wave activity (SWA), the electroencephalography power between 0.5 and $4 \mathrm{~Hz}$, during non-rapid eye movement sleep, is one of the best characterized markers of necessity of sleep. The main cause is that it increases as a function of preceding waking duration and decreases during sleep, but the underlying mechanisms remain unknown. A causal role for brain-derived neurotrophic factor in the homeostatic regulation of sleep has been observed [45-47]. BDNF has become increasingly accepted as a central mediator of the effects of stress on neuronal plasticity and its implication for psychopathology leading to neuropsychiatric diseases. The survival of neuron, learning, memory, appetite and sleep appear to be neurotropic functions of BDNF [45-47]. It is possible that neuro-cardiometabolic and stress-related mental disorders result from stress-induced decreases in BDNF expression. Disruption of sleep is a common feature in many stress-related mental disorders, which may predispose impairment of physical and mental health because sleep loss is often followed by higher stress vulnerability [48]. Insomnia is a common manifestation of depression among these patients [49] and in accordance with the neurotrophin hypothesis, BDNF may be involved in major depression and antidepressant effect $[50,51]$. Majority of workers have concentrated on specifying the role of BDNF in depression, the relation between BDNF and insomnia has been ignored. A recent study, investigated serum BDNF levels among 50 adults with symptoms of insomnia and healthy controls [44]. There was a significant difference in serum BDNF levels between smokers and non-smokers $(\mathrm{t}(47)=3.066 ; \mathrm{P}=$
0.004, ns: age, BMI and sex) as reported earlier [8]. Insomnia $(\mathrm{n}=26)$ exhibited significantly decreased serum BDNF levels compared with sleep-healthy controls $(n=24 ; \mathrm{F}(1)=$ 5.017; $\mathrm{P}=0.03$ ) Serum BDNF levels were also significantly correlated with severity of insomnia in all participants $(\mathrm{n}=$ $50 ; \mathrm{r}=-0.409 ; \mathrm{P}=0.004)$. While sleep impairment was associated with lower serum BDNF levels, good sleep was associated with higher serum BDNF levels. Serum BDNF levels were significantly lower in those reporting symptoms of fatigue $(\mathrm{n}=6)$ compared with sleep-healthy subjects $(\mathrm{n}=$ $6 ; \mathrm{t}=2.2625 ; \mathrm{P}=0.025)$ and were significantly correlated ( $\mathrm{n}$ $=12, \mathrm{r}=-0.639 ; \mathrm{P}=0.025)$ with the symptoms of tiredness and fatigue known to reflect malfunction of sleep. The findings support the hypothesis of increased stress vulnerability due to sleep loss, which may lead to decreased BDNF secretion. Such a decrease might be associated with a decrease in BDNF mRNA expression in peripheral blood mononuclear cells and/or may correspond to a decline of BDNF concentration in the brain. There is a need to examine association of peripheral BDNF levels with brain BDNF levels to find out its effects on gut hormones, cardio-myocyte and vascular endothelial functions. The association of serum BDNF concentration as potential indicator of brain alteration is justified, and a reduction of BDNF levels also linked to sleep disturbance [52]. However, wakefulness causing acute deprivation of sleep may be considered as a stressor for the brain which may cause adaptation leading to an increase in BDNF levels [53]. Therefore, one night of sleep deprivation, may be used as therapeutic intervention in some insomniac or depressed patients as a compensatory process to normalize BDNF levels [45]. There is some evidence that reduced peripheral BDNF mRNA levels are normalized by antidepressant treatment [54].

\section{BDNF AND ALCOHOLISM}

Several alterations in brain stress and reward systems have been reported after abstinence from chronic alcoholism. These adaptations include robust changes in the hypothalamic-pituitary-adrenal (HPA) and autonomic arousal pathways as well as in the mesolimbic dopamine system involved in the rewarding properties of alcohol. Serum BDNF level is variable in various reports, and interpretation is difficult due to limited information regarding analytical variance, biological variability, and the relative contribution of platelet and plasma pools to serum BDNF [55]. In a recent study, within- and between-subject variability, and group differences in serum and plasma BDNF, were assessed on three separate days in 16, 4-week abstinent alcohol dependent subjects $(7 \mathrm{M} / 9 \mathrm{~F})$ and 16 social drinkers (SDs; $8 \mathrm{M} / 8 \mathrm{~F})$. Significantly higher mean serum BDNF levels were observed for the alcohol dependent group compared to the social drinker group $(\mathrm{P}=0.003)$. No significant difference in mean baseline plasma BDNF levels was observed between the two groups. The low BDNF correlations that were observed between plasma and serum levels are congruent with their representing separate pools of BDNF [55]. The observation of higher basal serum BDNF in the alcohol dependent group without a concomitant elevation in plasma BDNF levels indicates that the elevated serum BDNF in these patients is not due to greater BDNF exposure. 


\section{BDNF, OBESITY AND INSULIN RESISTANCE}

BDNF is produced in brain neurons; hippocampus, cortex, basal forebrain, many nuclei in the brain stem, and in catecholamine neurons, including dopamine neurons in the substantia nigra. It is proposed that BDNF is also produced in the arcuate nucleus and pro-opiomelanocortin (POMC) neurons which are known to control food intake and weight gain as well as insulin release (Fig. 2). BDNF is also released in other tissues; salivary glands, stomach, duodenum, ileum, colon, lung, heart, liver, pancreas, kidney, bladder, uterus, oviduct, monocytes and eosinophils [57-59]. BDNF regulates functions of the gut and pancreatic beta islet activity in response to plasma levels of glucose, protein, fatty acids, insulin, and leptin in which hypothalamus plays a critical role. The food intake and body weight are regulated by BDNF. However, insulin resistance is determined by food intake and energy expenditure and weight gain which are under control of BDNF.

There is evidence that BDNF is also involved in all the inflammatory diseases; myocardial injury, rheumatological conditions, inflammatory bowel disease and atopic dermatitis, apart from insulin resistance, obesity, type 2 diabetes and metabolic syndrome [60-64]. BDNF deficiency observed in association with these diseases indicates that it may inhibit inflammation and inflammation can neutralize same causing a decrease in BDNF levels. Since the hypothalamus plays a critical role in energy homeostasis, it is important to identify factors that are relevant to glucose homeostasis. It seems that the arcuate nucleus metabolizes long chain fatty acids (LCFA) to produce long-chain fatty acyl-CoA intermediates (LCFACoA), which dampen appetite and reduce food intake leading to a decline in obesity and insulin resistance. Melanocortin 4 (MC4), melanocyte-stimulating hormone (MSH); POMC, pro-opiomelanocortin (POMC) neurons, neuropeptide-Y (NPY); and agouti-related protein (AGRP), appear to be important in this mechanism [1-4].

In experimental studies, treatment with BDNF decreased random blood glucose and body weight in obese, noninsulin-dependent diabetic C57BLKS-Lepr(db)/lepr(db) $(\mathrm{db} / \mathrm{db})$ mice and prevented diabetes [65-73]. The effects of BDNF on non-fasted blood glucose levels are not caused by the effects that persisted for weeks after cessation of BDNF treatment. It also reduced the hepatomegaly present in $\mathrm{db} / \mathrm{db}$ mice, in association with reduced liver glycogen and reduced liver enzyme activity in serum, supporting the involvement of liver tissue in the mechanism of action for BDNF [63]. BDNF administration, once or twice per week ( $70 \mathrm{mg} / \mathrm{kg} /$ week) to $\mathrm{db} / \mathrm{db}$ mice for 3 weeks significantly reduced blood glucose and hemoglobinA1c, $(\mathrm{HbA} 1 \mathrm{c}=$ glycosylated hemoglobin) as compared with control [23]. These results indicate that BDNF reduced blood glucose as well as restored systemic glucose balance and hence it can prevent insulin resistance.

The hypothalamic nuclei are associated with satiety and locomotor function, consisting of BDNF and its tyrosine kinase receptor expression, depending on food intake and sedentary behavior. BDNF gene expression is reduced in experimental mice and they exhibit abnormalities in eating behaviors and locomotor activity, which may be reversed by BDNF or neurotrophin 4/5 infusion [69]. Gene transfer of
BDNF can cause a marked reduction in weight and decrease in insulin resistance [69]. Experimental study indicates that BDNF activates the sympathetic nervous system via the central nervous system and regulates energy expenditure in obesity with diabetes [65]. In future, it may be possible that BDNF can be used as a hypoglycemic agent by administering it once in a week [66]. The decrease in hyperglycemia continuously for 7 days by administration of BDNF can provide better control of vascular complications of diabetes by decreasing superoxide anion, dyslipidemia, insulin resistance, free fatty acids, pro-inflammatory cytokines and endothelial dysfunction resulting in decline in CVDs. In an experimental model of obesity and type 2 diabetes [67], therapeutic efficacy of BDNF has been demonstrated. Gene transfer of BDNF may cause weight loss and alleviation of obesity and associated insulin resistance. BDNF plays a significant role in the regulation of appetite, obesity, and in the pathogenesis of insulin resistance by its action on hypothalamic neurons. BDNF may also modulate the release and activity of leptin, ghrelin, insulin, neuropeptide Y, melanocortin, serotonin, dopamine and other neuropeptides, neurotransmitters and gut hormones. It is possible that it would be a novel approach, in the management of metabolic syndrome, if BDNF can be delivered to the hypothalamus. PUFA has been found to have beneficial effects on obesity and type 2 diabetes which poses the possibility that both agents may have positive interactions. The combination of BDNF with w-3 fatty acids could be of additional benefit in the management of metabolic syndrome, because this complex of two agents would allow BDNF to pass the bloodbrain barrier easily, to reach the hypothalamus for its activity against metabolic syndrome [68]. W-3 fatty acids are present in large amounts in the brain and are known to increase the production of BDNF as they are natural enhancers of BDNF expression [68]. PUFA can also enhance the availability of endocannabinoid receptors which may be protective against obesity and metabolic syndrome [69].

There is evidence that peripheral inflammation induced an increased expression of BDNF mRNA which was mediated by nerve growth factor in the dorsal root ganglion. It is possible that BDNF might have a role in inflammation and hyperalgesia because nerve growth factor is a mediator of hyperalgesia. Experimental study using an intraplantar injection of Freund's adjuvant into rat paws caused significant increases in the percentage of BDNF-immunoreactive neuron profiles in the L5 dorsal root ganglion [70]. There was a marked increase in the expression of BDNF-IR terminals in the spinal dorsal horn following peripheral tissue inflammation produced [70]. These findings suggest that peripheral tissue inflammation induces an increased BDNF synthesis in the dorsal root ganglion and elevated anterograde transport of BDNF to the spinal dorsal horn.

\section{BDNF AND TYPE 2 DIABETES MELLITUS}

There is a low-grade systemic inflammation occurring in obesity, metabolic syndrome, CVDs and type 2 diabetes mellitus. Since BDNF is involved in their pathobiology, it is tempting to speculate that BDNF may also have a role in the regulation of inflammation and immune reactivity and brainheart interactions [56]. Experimental studies indicate that $\mathrm{BDNF}$ regulates functions of the gut and pancreatic beta islet 
activity in response to plasma levels of glucose, protein, fatty acids, insulin, and leptin in which hypothalamus plays a critical role [56, 71-73]. The food intake and body weight may be regulated by BDNF, present in the hippocampus, hypothalamus; arcuate nucleus and POMC neurons, cortex, basal forebrain, many nuclei in the brain stem, and catecholamine neurons, including dopamine neurons in the substantia nigra.

The above disorders of brain may be associated with type 2 diabetes, and animal models suggest that BDNF plays a role in insulin resistance. There is an inverse association between reduced BDNF concentration and type 2 diabetes and treatment with BDNF was associated with improvement in glucose metabolism, insulin sensitivity, and reduction in food consumption [26]. It is possible to conclude that BDNF may play an important role in the development of obesity and type 2 diabetes in humans. Plasma levels of BDNF were inversely associated with type 2 diabetes mellitus independent of obesity [64]. This study comprised of 233 subjects including; subjects with type 2 diabetes, obesity and healthy subjects. In Study 1, plasma levels of BDNF were decreased in patients with type 2 diabetes independently of obesity. Plasma BDNF was inversely associated with fasting plasma glucose, but not with insulin. No association was found between the BDNF G196A (Val66Met) polymorphism and diabetes or obesity. In Study 2, an output of BDNF from the human brain was detected at basal conditions. This output was inhibited when blood glucose levels were elevated. In contrast, when plasma insulin was increased while maintaining normal blood glucose, the cerebral output of BDNF was not inhibited, indicating that high levels of glucose, but not insulin, inhibit the output of BDNF from the human brain. It is possible that low levels of BDNF accompany impaired glucose metabolism. Decline in BDNF may be associated with dementia and depression, as well as with type 2 diabetes, potentially explaining the clustering of these conditions in epidemiological studies. A recent study also reported a significant inverse association between BDNF levels and $\mathrm{HbA} 1 \mathrm{c}$ indicating that BDNF may be used as a therapeutic agent for prevention and treatment of diabetes mellitus [10].

Experimental studies indicate that intracerebroventricular (ICV) administration of BDNF lowered blood glucose, increased pancreatic insulin content, enhanced thermogenesis and norepinephrine turnover, and increased uncoupling protein-1 mRNA expression in the interscapular brown adipose tissue of $\mathrm{db} / \mathrm{db}$ mice [65]. It is possible that BDNF activates the sympathetic nervous system via the central nervous system and regulates energy expenditure in obese diabetic animals. BDNF appears to be an anorexigenic factor that may be highly expressed in ventromedial hypothalamic (VMH) nuclei, arcuate nucleus and POMC neurons. The concentrations of BDNF in the brain are regulated by feeding status. It seems that stress hormone corticosterone decreases the expression of BDNF in rats and may cause an eventual atrophy of the hippocampus, suggesting that BDNF and neurons in the brain play a critical role in obesity and type 2 diabetes mellitus [28]. Circulating insulin acts as an adiposity signal to the brain, especially on the arcuate nucleus of the hypothalamus, which, in turn, controls energy homeostasis [50]. Insulin stimulates the synthesis of POMC, which acts on melanocortin receptors MC3R and MC4R in several hypo- thalamic nuclei [50]. Tamura et al. [74] reported that ghrelin stimulates $\mathrm{GH}$ but no food intake in arcuate nucleus ablated rats, indicating the further importance of the arcuate nucleus in the hypothalamus.

Associations among plasma BDNF, cognition, and insulin function were studied among 41 subjects with impaired insulin function, ranging from insulin resistance to type 2 diabetes mellitus [26]. These patients were matched with 41 healthy controls on gender, age, education, and IQ levels. Individuals with impaired insulin function had significantly lower plasma BDNF levels than controls, particularly females, and higher BDNF levels were associated with poorer explicit memory in these females. It is possible that higher BDNF levels within this group may reflect the body's efforts to respond to damage. After accounting for age, education, and $\mathrm{HbA} 1 \mathrm{c}, \mathrm{BDNF}$ significantly predicted $13.1-23.5 \%$ of the variance in explicit memory in women. The results indicate that BDNF elevations within diseased groups may not always be a marker of health but may be due to compensatory mechanisms.

Suwa et al. also reported that serum BDNF levels are increased in patients associated with obesity in newly diagnosed female patients with type 2 diabetes mellitus [75]. In this study, BDNF levels were measured in the serum among 24 patients, aged 34-56 years, in newly diagnosed female patients with type 2 diabetes mellitus and female subjects with normal glucose tolerance ( $n=7$, aged $34-56$ years). The serum BDNF levels were found to significantly increase in diabetic patients in comparison to those in healthy subjects $(\mathrm{P}<0.05)$. The serum BDNF levels showed positive correlation with the body mass index, the percentage of body fat, the subcutaneous fat area based on computed tomography scan, the triglyceride level, the fasting blood glucose level, and the homeostasis model assessment of insulin resistance score. BDNF levels showed a negative correlation with age. The partial correlation coefficients adjusted by age showed significant differences regarding the body mass index $(\mathrm{r}=$ $0.423, \mathrm{P}<0.05)$, percentage of body fat $(\mathrm{r}=0.504, \mathrm{P}<0.05)$, and triglyceride levels $(\mathrm{r}=0.426, \mathrm{P}<0.05)$. These results provide the first evidence that an increased BDNF is associated with a prevalence of type 2 diabetes mellitus. The BDNF was related to the total and abdominal subcutaneous fat mass and energy metabolism in the newly diagnosed female patients with type 2 diabetes mellitus.

\section{BDNF AND CARDIOVASCULAR DISEASES}

NCDs, particularly CVDs and type 2 diabetes have become a major cause of mortality in most of the countries of the world [76, 77]. Vascular endothelial growth factor (VEGF), IL-6, and clusterin have important role in neuroprotection as well as vascular diseases [78]. Low circulating BDNF concentrations have been observed in patients with coronary artery disease, type 2 diabetes mellitus, metabolic syndrome, acute coronary syndrome, and physical inactivity $[79,80]$. There is evidence indicating higher VEGF concentrations in patients with hypertension, type 2 diabetes mellitus, smoking, obesity, and coronary artery disease, all risk factors associated with stroke [80-83]. Experimental studies showed that under hypoxic and ischemic conditions, both BDNF and VEGF and their respective receptors are upregulated in neurons and vascular endothelial cells [84-86]. 
Therapeutic angiogenesis strategy may be of high potential for the regeneration of the infarcted myocardium to prevent cardiovascular mortality and morbidity after myocardial infarction. Recently, it has been reported that BDNF and its receptor, TrkB, are expressed first during late gestation and then persistently at high levels into adulthood in the endothelial cells lining, the arteries and capillaries of the heart [86]. The number of truncated TrkB-positive cells and BDNF protein levels are higher in the aged cardiac microvasculature. BDNF overexpression in the mid-gestational mouse heart results in an increase in capillary density. Regarding exogenous delivery of basic fibroblast growth factor (bFGF) and BDNF in ischemic myocardium, BDNF may improve the angiogenesis and left ventricular function of the ischemic myocardium compared with bFGF [87]. After myocardial infarction, BDNF expression is up-regulated by neural signals from the young heart and then protects the myocardium against ischemic injury [88]. BDNF levels in aged ischemic cardiac microvasculature are higher compared with levels in young non-ischemic, young ischemic and old non-ischemic hearts. The age-related increase in truncated TrkB in cardiac microvascular endothelial cells is apparently linked to an age-associated increase in inflammatory response and a significant increase in myocardial injury following coronary artery occlusion. It is known that the BDNF-TrkB pathway plays an important role in the development of cardiac vasculature, cardiac protection, angiogenesis of the myocardium and myocardial injury seen in the aged heart.

Pikula et al. examined the association of serum BDNF and VEGF with the risk of clinical stroke or subclinical vascular brain injury in a community-based sample of 3440 Framingham Study participants (mean age, 65 \pm 11 years; $56 \%$ women) who were free from stroke/transient ischemic attack (TIA) [89]. After a follow up of 10 years, 193 participants experienced incident stroke/TIA. In multivariable analyses, lower BDNF and higher log VEGF levels were associated with an increased risk of incident stroke/TIA. Lower serum BDNF and higher VEGF concentrations were associated with increased risk of incident stroke/TIA. Higher levels of BDNF were also associated with less white matter hyper intensity and better visual memory. These results indicate that circulating BDNF and VEGF levels modify risk of clinical and subclinical vascular brain injury. It seems that BDNF and VEGF are novel risk markers and may improve stratification of patients at risk for stroke/TIA. As a marker of neuronal function, BDNF may serve as an intermediate biomarker for subclinical vascular disease. BDNF may have biological potential to serve as a therapeutic target for primary and secondary prevention of cardio-metabolic risk as well as clinical and subclinical vascular brain disease.

\section{NUTRITIONAL FACTORS AND BDNF}

There is evidence that insulin resistance may be associated with increased risk for developing cognitive decline and neuro-degeneration, vascular dementia, depression and metabolic syndrome, which is a risk factor for type 2 diabetes and CVDs. Low plasma BDNF levels were observed in neurodegenerative diseases as well as in obesity and type 2 diabetes. A decrease in serum BDNF concentration in young non-obese subjects can occur with low insulin sensitivity. Insulin resistance might play a role in common pathogenesis of neurodegenerative and metabolic disorders, and decreased BDNF may explain the clustering of these diseases leading to CVDs and type 2 diabetes. The mechanism connecting insulin resistance with neuro-degeneration is still unclear but it is widely accepted that free fatty acids (FFA) induce insulin resistance. It is noteworthy that lack of exercise and a high-fat diet (HFD) disrupt cognition and contribute to neurodegenerative diseases $[90,91]$. A recent study, among 18 obese young adult males and 20 healthy males, examined the effects of hyperinsulinemia and serum free fatty acids (FFA) elevation on circulating BDNF concentration in humans [91]. Insulin sensitivity was reduced by $\sim 40 \%$ after $6 \mathrm{~h}$ of intralipid/heparin infusion $(\mathrm{P}<0.001)$. During both clamps, i.e. euglycemic hyperinsulinemic clamp with or without intralipid/heparin infusion, serum and plasma BDNF followed the same pattern. Hyperinsulinemia had no effect on circulating BDNF. Raising FFA had no effect on circulating BDNF in $120 \mathrm{~min}$; however, it resulted in a significant decrease by $43 \%$ in serum and by $35 \%$ in plasma BDNF after 360 min ( $P$ $=0.005$ and 0.006 , respectively). High-fat meal also resulted in a decrease by $27.8 \%$ in plasma BDNF $(\mathrm{P}=0.04)$. These results indicate that increasing free fatty acids in the blood decreases circulating BDNF. This might indicate a potential link between free fatty acid-induced insulin resistance and neurodegenerative disorders.

The main source of circulating BDNF in response to exercise is not known. In a recent study, eight volunteers rowed for $4 \mathrm{~h}$ while simultaneous blood samples were obtained from the radial artery and the internal jugular vein, to investigate the role of exercise in BDNF release [90]. Mouse brains were dissected and analyzed for BDNF mRNA expression following treadmill exercise to identify putative cerebral region(s) responsible for BDNF release. BDNF release from the brain was observed at rest $(\mathrm{P}<0.05)$, and was found to have increased two- to three-fold during exercise $(\mathrm{P}<0.05)$ in humans. Both at rest and during exercise, the brain contributed $70-80 \%$ of circulating BDNF, while that contribution decreased following $1 \mathrm{~h}$ of recovery. Exercise induced a three- to five-fold increase in BDNF mRNA expression in the hippocampus and cortex, peaking $2 \mathrm{~h}$ after the termination of exercise in mice. These results suggest that the brain is a major but not the sole contributor to circulating BDNF. Moreover, the importance of the cortex and hippocampus as a source for plasma BDNF becomes even more prominent in response to exercise.

The relationship of BDNF with cardio-metabolic risk factors is unclear, and the effect of exercise training on BDNF has not been previously explored in individuals with type 2 diabetes [27]. This study comprised of 150 women with type 2 diabetes who were randomized to an aerobic exercise (aerobic), resistance exercise (resistance), or a combination of both (combination) for 9 months. Baseline serum BDNF was not associated with fitness, body composition, anthropometry, glucose control, or strength measures (all, $\mathrm{P}>0.05$ ). Similarly, no significant change in serum BDNF levels was observed following exercise training in the aerobic, resistance, or combination groups compared to the control group. However, reductions in waist circumference were directly associated with changes in serum BDNF following training $(\mathrm{r}=0.25, \mathrm{P}=0.005)$. The findings indicated that, serum BDNF was not associated with fitness, body composition, 
anthropometry, glucose control, or strength measures at baseline. Likewise, serum BDNF measures were not altered by 9 months of aerobic, resistance, or combination training. However, reductions in waist circumference were associated with decreased serum BDNF levels. Future studies should investigate the relevance of BDNF with measures of cognitive function specifically in individuals with type 2 diabetes.

Dietary restriction increases the number of newly generated neuronal cells, induces BDNF expression in the dentate gyrus of rats [92], and increases serum BDNF in humans [93]. Physical activity increases BDNF measured both in serum and in plasma [92, 94]. BDNF might also regulate glucose metabolism but the hypoglycemic effect of BDNF cannot be ascribed solely to the hypophagic effect of BDNF, because BDNF administration improves insulin resistance in $d b / d b$ mice, even when food intake is controlled. BDNF is abundantly expressed in central and peripheral nervous system and can cross the blood-brain barrier in both directions. Further studies have shown that BDNF is also produced in non-neurogenic tissues, including skeletal muscle and vascular endothelium, and is stored in platelets which pose the possibility that BDNF can influence platelet dysfunction and thrombosis. Because serum contains the factors released from the platelets, it could be an important issue whether BDNF concentration is measured in serum or in plasma. The changes in plasma BDNF are considered to reflect its changes in the brain. However, serum BDNF concentration has also been reported to closely correlate with the cortical BDNF level, indicating that it can reflect the BDNF level in the brain tissue. Rasmussen et al. reported that the brain contributes to approximately $70-80 \%$ of plasma BDNF in healthy humans [90]. It is known that low levels of BDNF are associated with reduced function of alpha2/delta- 1 protein in the hypothalamus which facilitates the function of BDNF. BDNF is known to suppress appetite but the exact mechanism how BDNF inhibits appetite and overeating is yet unknown [95]. This study has proven that alpha2/delta-1 protein is necessary for normal BDNF function which could be a new target for treatment of obesity [95].

In brief, $\mathrm{BDNF}$ is a key protein in regulating neuronal survival, differentiation, and synaptic plasticity and seems important for learning and memory function due to its specific effects on central pathways involved in appetite regulation and energy expenditure. Blood BDNF levels were inversely associated with anxiety, depression, insomnia, obesity, insulin resistance, metabolic syndrome, type 2 diabetes and stroke. Okada et al. have observed that BDNF protects against cardiac dysfunction after myocardial infarction via central nervous system mediated pathway [88]. BDNF deficiency increased appetite resulting into overeating and obesity. It is proposed that high fat diet and rapidly absorbed fast foods may cause increase in free fatty acids and insulin resistance with a decrease in BDNF levels and exercise may increase BDNF levels causing beneficial effects on BDNF and nerve growth factor. Randomized, controlled trials and cohort studies would be necessary to demonstrate the role of BDNF on CVDs, type 2 diabetes and in depressive disorders.

\section{CONFLICT OF INTEREST}

The author(s) confirm that this article content has no conflicts of interest.

\section{ACKNOWLEDGEMENTS}

Thanks are paid to International College of Nutrition and International College of Cardiology and the Tsim Tsoum Institute for logistic support to write this article.

\section{REFERENCES}

[1] Pella D, Singh RB, Otsuka K, Chiang CE, Joshi SR. Nutritional predictors and modulators of metabolic syndrome. J Nutr Environ Med 2004; 14: 3-163.

[2] Pella D, Thomas N, Tomlinson B, Singh RB. Prevention of coronary artery disease: the South Asian paradox. Lancet 2003; 361: 79 .

[3] Dubnov Gal, Pella D, Singh RB. The effect of an alpha-linolenic acid rich diet on the circadian rhythm of cardiac events. World Heart J 2008; 1: 49-56.

[4] Singh RB, Pella D, Otsuka K. Can brain dysfunction be a predisposing factor in metabolic syndrome? Biomed Pharmacother 2004; 58: 52-68.

[5] Pella D, Otsuka K, Singh RB. Metabolic Syndrome: A disease of the brain. Open Nutra J 2011; 4: 107-18.

[6] Singh RB, Cornelissen G, Weydahl A, et al. Circadian heart rate and blood pressure variability considered for research and patient care. Int J Cardiol 2003; 87: 9-28.

[7] Singh RB, Weydahl K, Otsuka K, et al. Can nutrition influence heart rate and blood pressure variability. Biomed Pharmacother 2001; 55: 7-18.

[8] Borovikova LV, Ivanova S, Zhang M, et al. Vagal nerve stimulation attenuates the systemic inflammatory response to endotoxin. Nature 2000; 405: 458-62.

[9] Singh RB, Gupta S, Dherange P, et al. Metabolic syndrome: a brain disease. Can J Physiol Pharmacol 2012; 90: 1171-83.

[10] Yoshida T, Ishikawa M, Niitsu T, et al. Decreased serum levels of mature Brain-Derived Neurotrophic Factor (BDNF), but not its precursor proBDNF, in patients with major depressive disorder. PLoS One 2012; 7(8): e42676.

[11] Halberg F, Cornélissen G, Czaplicki J, et al. Beyond a spotcheckbased health care: Thank you, Ram Bahadur Singh. Open Nutr J 2011; 4: 34-44.

[12] Suliman S, Hemmings MJ, Seedat S. Brain-derived neurotrophic factor (BDNF) protein levels in anxiety disorders: systematic review and meta-regression analysis. Front Integr Neurosci 2013 doi: 10.3389/fnint.2013.00055

[13] Ali S, Stone MA, Peters JL, Davies MJ, Khunti K. The prevalence of co-morbid depression in adults with type 2 diabetes: a systematic review and meta-analysis. Diabet Med 2006; 23: 1165-73.

[14] Feinkohl I, Sattar N, Welsh P, Reynolds RM, Deary IJ. Association of N-terminal pro-brain natriuretic peptide with cognitive function and depression in elderly people with type 2 diabetes. PLoS One 2012; 7: e44569.

[15] Egede LE, Nietert PJ, Zheng D. Depression and all-cause and coronary heart disease mortality among adults with and without diabetes. Diabetes Care 2005; 28: 1339-45.

[16] Bogner HR, Morales KH, Post EP, Bruce ML. Diabetes, depression, and death: a randomized controlled trial of a depression treatment program for older adults based in primary care (PROSPECT]. Diabetes Care 2007; 30: 3005-10.

[17] Mattson MP, Maudsley S, Martin B. BDNF and 5-HT: a dynamic duo in age-related neuronal plasticity and neurodegenerative disorders. Trends Neurosci 2004; $27:$ 589-94.

[18] Tsai SJ, Hong CJ, Liou YJ. Effects of BDNF polymorphisms on antidepressant action. Psychiatry Investig 2010; 7: 236-42.

[19] Duman RS. Role of neurotrophic factors in the etiology and treatment of mood disorders. Neuromol Med 2004, 5(1): 11-25.

[20] Karege F, Perret G, Bondolfi G, Schwald M, Bertschy G. Decreased serum brain-derived neurotrophic factor levels in major depressed patients. Psychiatry Res 2002; 109: 143-8.

[21] Sen S, Duman R, Sanacora G. Serum brain-derived neurotrophic factor, depression, and antidepressant medications: meta-analyses and implications. Biol Psychiatry 2008, 64(6): 527-32.

[22] Duman RS. Structural alterations in depression: cellular mechanisms underlying pathology and treatment of mood disorders. CNS Spectr 2002; 7: 140-2.

[23] Kernie SG, Liebl DJ, Parada LF. BDNF regulates eating behavior and locomotor activity in mice. EMBO J 2000; 19(6): 1290-300. 
[24] Fujinami A, Ohta K, Obayashi H, Fukui M, Hasegawa G, Nakamura N. Serum brain-derivedneurotrophic factor in patients with type 2 diabetes mellitus: Relationship to glucose metabolism and biomarkers of insulin resistance. Clin Biochem 2008; 41(10-11): 812-7.

[25] Rao AA, Sridhar GR, Srinivas B, Das UN. Bioinformatics analysis of functional protein sequences reveals a role for brain-derived neurotrophic factor in obesity and type 2 diabetes mellitus. Med Hypotheses 2008; 70(2): 424-9.

[26] Arentoft A, Sweat V, Starr V, Oliver S, Hassenstab J. Plasma BDNF is reduced among middle-aged and elderly women with impaired insulin function: evidence of a compensatory mechanism. Brain Cogn 2009; 71: 147-52.

[27] Swift DL, Johannsen NM, Myers VH, Earnest CP, Smits JA. The effect of exercise training modality on serum brain derived neurotrophic factor levels in individuals with type 2 diabetes. PLoS One 2012; 7(8): e42785.

[28] Das UN. Is type 2 diabetes mellitus a disorder of the brain. Nutrition $2002 ; 18$ : 667-72.

[29] Engum A. The role of depression and anxiety in onset of diabetes in a large population-based study. J Psychosom Res 2007; 62(1): $31-8$.

[30] Ventriglia M, Chiavetto BL, Benussi L, Binetti G, Zanetti O. Association between the BDNF $196 \mathrm{~A} / \mathrm{G}$ polymorphism and sporadic Alzheimer's disease. Mol Psychiatry 2002; 7(2): 136-7.

[31] Egan MF, Kojima M, Callicott JH, Goldberg TE, Kolachana BS. The BDNF val66met polymorphism affects activity-dependent secretion of BDNF and human memory and hippocampal function. Cell 2003; 112: 257-69.

[32] Molendijk ML, van Tol MJ, Penninx BW, van der Wee NJ, Aleman A, Veltman DJ. BDNF val66met affects hippocampal volume and emotion-related hippocampal memory activity. Transl Psychiatry 2012;31(2): e74.

[33] Ribeiro L, Busnello JV, Cantor RM, Whelan F, Whittaker P. The brain-derived neurotrophic factor rs6265 (Val66Met) polymorphism and depression in Mexican-Americans. Neuroreport 2007; 18: 1291-3.

[34] Jiang X, Xu K, Hoberman J, Tian F, Marko AJ. BDNF variation and mood disorders: a novel functional promoter polymorphism and Val66Met are associated with anxiety but have opposing effects. Neuropsychopharmacology 2005; 30(7): 1353-61.

[35] Schumacher J, Jamra RA, Becker T, Ohlraun S, Klopp N. Evidence for a relationship between genetic variants at the brain-derived neurotrophic factor (BDNF) locus and major depression. Biol Psychiatry 2005; 58: 307-14.

[36] Carrard A, Salzmann A, Perroud N, Gafner J, Malafosse A, Karege F. Genetic association of the Phosphoinositide-3 kinase in schizophrenia and bipolar disorder and interaction with a BDNF gene polymorphism. Brain Behav 2011; 1(2): 119-24.

[37] Zhou JX, Li HC, Bai JX et al. Functional Val66Met polymorphism of Brain-derived neurotrophic factor in type 2 diabetes with depression in Han Chinese subjects. Behav Brain Funct 2013; 9: 34

[38] Tsai SJ, Hong CJ, Liou YJ. Effects of BDNF polymorphisms on antidepressant action. Psych Investig 2010; 7(4): 236-42.

[39] Outhred T, Das P, Dobson-Stone C, Griffiths K, Felmingham KL, Bryant RA. The functional epistasis of 5-HTTLPR and BDNF Val66Met on emotion processing: a preliminary study. Brain Behav 2012; 2(6): 778-88.

[40] Vasant DH, Payton A, Mistry S, Thompson DG, Hamdy S. The val66met polymorphism of brain-derived neurotrophic factor is associated with human esophageal hypersensitivity. Neurogastroenterol Motil 2013; 25(2): 162-e85.

[41] Smolders R, Rijpkema M, Franke B, Fernández G. BDNF Val66Met polymorphism interacts with sex to influence bimanual motor control in healthy humans. Brain Behav 2012; 2: 726-31.

[42] Hwang JP, Tsai SJ, Hong CJ, Yang CH, Lirng JF. The Val66Met polymorphism of the brain-derived neurotrophic-factor gene is associated with geriatric depression. Neurobiol Aging 2006; 27: 1834-7.

[43] Hong CJ, Huo SJ, Yen FC, Tung CL, Pan GM, Tsai SJ. Association study of a brain-derived neurotrophic-factor genetic polymorphism and mood disorders, age of onset and suicidal behavior. Neuropsychobiology 2003; 48: 186-9.

[44] Chen ZY, Jing D, Bath KG, Ieraci A, Khan T. Genetic variant BDNF (Val66Met) polymorphism alters anxiety-related behavior. Science 2006; 314: 140-3.
[45] Giese M, Unternährer E, Hüttig H, et al. BDNF: an indicator of insomnia? Mol Psychiatry 2014; 19(2): 151-2.

[46] Duman RS, Malberg J, Nakagawa S, D'Sa C. Neuronal plasticity and survival in mood disorders. Biol Psychiatry 2000; 48: 732-7.

[47] Faraguna U, Vyazovskiy VV, Nelson AB, Tononi G, Cirelli C. A causal role for brain-derived neurotrophic factor in the homeostatic regulation of sleep. J Neurosci 2008; 28: 4088-95.

[48] Steiger A, Kimura M. Wake and sleep EEG provide biomarkers in depression. J Psychiatr Res 2010; 44: 242-52.

[49] Molendijk ML, Bus BA, Spinhoven P, Penninx BW, Kenis G, Prickaerts J. BDNF as biomarker for depression. Mol Psychiatry 2011; 16: 1088-10.

[50] Lang UE, Hellweg R, Gallinat J. BDNF serum concentrations in healthy volunteers are associated with depression related personality traits. Neuropsychopharmacology 2004; 29: 795-8.

[51] Bus BA, Molendijk ML, Penninx BJ, et al. Determination of serum brain derived neurotrophic factor. Psychoneuroendocrinology 2011; 36: 228-39.

[52] Sartorius A, Hellweg R, Litzke J, et al. Correlations and discrepancies between serum and brain tissue levels of neurotrophins after electroconvulsive treatment in rats. Pharmacopsychiatry 2009; 42: 270-2.

[53] Conti B, Maier R, Barr AM, et al. Region-specific transcriptional changes following the three antidepressant treatments electro convulsive therapy, sleep deprivation and fluoxetine. Mol Psychiatry 2007; 12: 167-89.

[54] Cattaneo A, Bocchio-Chiavetto L, Zanardini R, Milanesi E, Placentino A, Gennarelli M. Reduced peripheral brain-derived neurotrophic factor mRNA levels are normalized by antidepressant treatment. Int J Neuropsychopharmacol 2010; 13: 103-8.

[55] D'Sa C, Dileone RJ, Anderson GM, Sinha R. Serum and plasma brain-derived neurotrophic factor (BDNF) in abstinent alcoholics and social drinkers. Alcohol 2012; 46(3): 253-2.

[56] Singh RB, Chaudhuri J, De Meester F, Wilzynska A, Wilson DW, Hungin APS. The gut, brain and heart connection. World Heart J 2011; 3: 151-74.

[57] Lommatzsch M, Braun A, Mannsfeldet A, et al. Abundant production of brain derived neurotrophic factor by adult visceral epithelia Am J Pathol 1999; 155: 1183-93.

[58] Rost B, Hanf G, Ohnemus U. Monocytes of allergics and nonallergics produce, store and release the neurotrophins NGF, BDNF and NT. Regul Pept 2005; 124: 19-25.

[59] Noga O, Englmann C, Hanf G, Grützkau A, Seybold J, Kunkel G. The production, storage and release of the neurotrophins nerve growth factor, brain-derived neurotrophic factor and neurotrophins3 by human peripheral eosinophils in allergics and non-allergics. Clin Exp Allergy 2003; 33: 649-54.

[60] Cai D, Holm JM, Duignan IJ, Zheng J, Xaymardan M, Chin A. BDNF-mediated enhancement of inflammation and injury in the aging heart. Physiol Genomics 2006; 24: 191-7.

[61] Johansson M, Norrgard O, Frogmen S. Study of expression patterns and levels of neurotrophins and neurotrophin receptors in ulcerative colitis. Inflamm Bowel Dis 2007; 13: 398-409.

[62] di Mola F, Friess H, Zhu ZW, et al. Nerve growth factor and Trk high affinity receptors (TrkA) gene expression in inflammatory bowel disease. Gut 2000; 46: 670-9.

[63] Raap U, Werfel T, Goltz C, et al. Circulating levels of brainderived neurotrophic factor correlate with disease severity in the intrinsic type of atopic dermatitis. Allergy 2006; 61: 1416-8.

[64] Krabbe KS, Nielsen AR, Krogh-Madsen R, et al. Brain derived neurotrophic factor and type 2 diabetes. Diabetologia 2007; 50: 431-38.

[65] Nonomura T, Tsuchida A, Ono-kishino M, Nakagawa T, Taiji M, Noguchi H. Brain derived neurotrophic factor regulates energy expenditure through the central nervous system in obese diabetic mice. Int J Exp Diab Res 2001; 2: 201-9.

[66] Ono M, Itakura Y, Nonomura T. Intermittent administration of brain derived neurotrophic factor ameliorates glucose metabolism in the obese diabetic mice. Metabolism 2000; 49: 129-33.

[67] Cao L, D Lin EJ, Cahill MC, Wang C, Liu X, During MJ. Molecular therapy of obesity and diabetes by a physiological autoregulatoryapproach. Nat Med 2009; 15: 447-54.

[68] Rao JS, Ertley RN, JLee H, et al. n-3 polyunsaturated fatty acid deprivation in rats decreases frontal cortex BDNF via a p38 MAPK-dependent mechanism. Mol Psychiatry 2007; 12: 36-6. 
[69] Di Marzo V. The endocannabinoid system in obesity and type 2 diabetes. Diabetologia 2008; 51: 1356-67.

[70] Cho HJ, Kim JK, Zhou XF, Rush RA. Increased brain derived neurotrophic factor immunoreactivity in rat dorsal root ganglia and spinal cord following peripheral inflammation. Brain Res 1997; 764: 269-72.

[71] Komori T, Morikawa Y, Nanjo K, Senba E. Induction of brainderived neurotrophic factor by leptin in the ventromrdial hypothalamus. Neuroscience 2006; 139: 1107-15.

[72] Tonra JR, Ono M, Liu X, Garcia K, Jackson C. Brain derived neurotrophic factor improves blood glucose control and alleviates fasting hyperglycemia in C57BLKS-lepr(db)mice. Diabetes 1999; 48: 588-94.

[73] Nakagawa T, Ogawa Y, Ebihara K, et al. Antiobesity and antidiabetic effects of brain derived neurotrophic factor in rodent models of leptin resistance. Int J Obes Rel Meta Disorder 2003; 27: 55765 .

[74] Tamura H, Kamegai J, Shimizu T, Ishii S, Sugihara H, Oikawa S. Ghrelin stimulates growth hormones but not food intakes in arcuate nucleus ablated rats. Endocrinology 2002; 143: 3268-75.

[75] Suwa M, Kishimoto H, Nofuji Y, et al. Serum brain-derived neurotrophic factor level is increased and associated with obesity in newly diagnosed female patients with type 2 diabetes mellitus. Metabolism 2006; 55(7): 852-7.

[76] WHO. Mortality and burden of disease estimates for WHO Member States in 2008. Geneva: World Health Organization 2010

[77] Editorial. Wealth but not health in USA. Lancet 2013; 381: 177.

[78] Pucci S, Mazzarelli P, Missiroli F, Regine F, Ricci F. Neuroprotection:VEGF, IL-6, and clusterin: the dark side of the moon. Prog Brain Res 2008; 173: 555-73.

[79] Golden E, Emiliano A, Maudsley S, Windham BG, Carlson OD, Egan JM. Circulating brain-derived neurotrophic factor and indices of metabolic and cardiovascular health: data from the Baltimore Longitudinal Study of Aging. PLoS One 2010; 5: e10099.

[80] Ferris LT, Williams JS, Shen CL. The effect of acute exercise on serum brain-derived neurotrophic factor levels and cognitive function. Med Sci Sports Exerc 2007; 39: 728-34.

[81] Blann AD, Belgore FM, McCollum CN, Silverman S, Lip PL, Lip GY. Vascular endothelial growth factor and its receptor, Flt-1, in the plasma of patients with coronary or peripheral atherosclerosis, or Type II diabetes. Clin Sci (Lond) 2002; 102: 187-94.

[82] Lieb W, Safa R, Benjamin EJ, Xanthakis V, Yin X, Sullivan LM. Vascular endothelial growth factor, its soluble receptor, and hepatocyte growth factor: clinical and genetic correlates and association with vascular function. Eur Heart J 2009; 30: 1121-7.
[83] Wolf PA, D'Agostino RB, Belanger AJ, Kannel WB. Probability of stroke: a risk profile from the Framingham Study. Stroke 1991; 22: 312-8.

[84] Lennmyr F, Ata KA, Funa K, Olsson Y, Terént A. Expression of vascular endothelial growth factor (VEGF) and its receptors (Flt-1 and Flk-1) following permanent and transient occlusion of the middle cerebral artery in the rat. J Neuropathol Exp Neurol 1998; 57: 874-82.

[85] Santhanam AV, Smith LA, Katusic ZS. Brain-derived neurotrophic factor stimulates production of prostacyclin in cerebral arteries. Stroke 2010; 41: 350-6.

[86] Cao L, Zhang L, Chen S, et al. BDNF-mediated migration of cardiac microvascular endothelial cells is impaired during ageing. $\mathrm{J}$ Cell Mol Med 2012; 16: 3105-15.

[87] Liu Y, Sun L, Huan Y, et al. Application of bFGF and BDNF to improve angiogenesis and cardiac function. J Surg Res 2006; 136 : 85-91.

[88] Okada S, Yokoyama M, took $\mathrm{H}$ et al. BDNF protects against cardiac dysfunction after myocardial infarction via central nervous system mediated pathway. Arterioscler Thromb Vasc Biol 2012; 32: 1902-2009.

[89] Pikula A, Beiser AS, Chen TC, et al. Serum brain-derived neurotrophic factor and vascular endothelial growth factor levels are associated with risk of stroke and vascular brain injury. Framingham Study. Stroke 2013; 44: 2768-75.

[90] Rasmussen P, Brassard P, Adser H, et al. Evidence for a release of brain-derived neurotrophic factor from the brain during exercise. Exp Physiol 2009; 94: 1062-9.

[91] Karczewska-Kupczewska M, Kowalska I, Nikołajuk A, et al. Circulating brain-derived neurotrophic factor concentration is down regulated by intralipid/heparin infusion or high-fat meal in young healthy male subjects. Diabetes Care 2012; 35: 358-62.

[92] Lee J, Duan W, Long JM, Ingram DK, Mattson MP. Dietary restriction increases the number of newly generated neural cells, and induces BDNF expression, in the dentate gyrus of rats. J Mol Neurosci 2000; 15: 99-108.

[93] Araya AV, Orellana X, Espinoza J. Evaluation of the effect of caloric restriction on serum BDNF in overweight and obese subjects: preliminary evidences. Endocrine 2008; 33: 300-3.

[94] Tang SW, Chu E, Hui T, Helmeste D, Law C. Influence of exercise on serum brain-derived neurotrophic factor concentrations in healthy human subjects. Neurosci Lett 2008; 431: 62-5.

[95] Cordeira JW, Felsted JA, Teillon S, et al. Hypothalamic dysfunction of the thrombospondin receptor $\alpha 2 \delta-1$ underlies the overeating and obesity triggered by BDNF deficiency. J Neurosci 2014; 34(2): $554-65$.

(C) Singh et al.; Licensee Bentham Open.

This is an open access article licensed under the terms of the Creative Commons Attribution Non-Commercial License (http://creativecommons.org/licenses/by-nc/3.0/) which permits unrestricted, non-commercial use, distribution and reproduction in any medium, provided the work is properly cited. 